\title{
An Unusual Cause of Lumbar Radiculopathy
}

\author{
Jesse Even MD, Gregory Gasbarro MD, \\ Liron Pantanowitz MD, James Kang MD, \\ Kurt Weiss MD
}

Received: 24 November 2014 / Accepted: 24 March 2015/Published online: 10 April 2015

(C) The Association of Bone and Joint Surgeons ( 2015

\section{History and Physical Examination}

A 46-year-old man with no significant medical or family history was referred to our tertiary spine surgery clinic for evaluation. He had been having low back pain and right lower extremity pain originating in his posterior thigh and radiating to his foot since lifting some heavy equipment approximately 1 year before his presentation to us. He originally sought treatment with a chiropractor, which did not alleviate his symptoms. He then presented to his primary care physician, who ordered physical therapy, which the patient completed without any relief of his back or leg

Each author certifies that he or she, or a member of his or her immediate family, has no funding or commercial associations (eg, consultancies, stock ownership, equity interest, patent/licensing arrangements, etc) that might pose a conflict of interest in connection with the submitted article.

All ICMJE Conflict of Interest Forms for authors and Clinical Orthopaedics and Related Research ${ }^{\circledR}$ editors and board members are on file with the publication and can be viewed on request.

Each author certifies that his or her institution waived approval for the reporting of this case and that all investigations were conducted in conformity with ethical principles of research.

J. Even, G. Gasbarro, J. Kang, K. Weiss ( $₫)$

Department of Orthopaedic Surgery, University of Pittsburgh Medical Center, Shadyside Medical Building, 5200 Centre Ave.,

Suite 415, Pittsburgh, PA 15232, USA

e-mail:weiskr@upmc.edu; weisskr@upmc.edu

L. Pantanowitz

Department of Pathology, University of Pittsburgh Medical

Center, Pittsburgh, PA, USA pain. His primary care physician then ordered MRI of the lumbar spine and sent the patient for epidural steroid injections, which again did not provide any pain relief of low back or right lower extremity pain. The patient also began a course of gabapentin (600 mg orally, three times daily). On further questioning at our clinic, the patient stated that he was being treated for a hamstring strain by his primary care physician ipsilateral to his radicular symptoms for several months. He claimed that the discomfort was exacerbated by riding on his lawnmower or sitting in the car.

Physical examination revealed normal strength of the right lower extremity, decreased sensation along the plantar heel and lateral foot, and decreased Achilles reflex. Examination of his thigh in the prone position showed a large, palpable mass in his deep soft tissues and a positive Tinel's sign, which radiated to his foot on palpation. We reviewed his previous MR images of the lumbar spine and subsequently ordered plain radiographs (Fig. 1), and MRI with gadolinium contrast of the right lower extremity for further evaluation (Figs. 2, 3). Based on the patient's history, physical examination, and imaging studies, what is the differential diagnosis at this point?

\section{Imaging Interpretation}

Prior MRI of the lumbar spine from the outside hospital showed no obvious spinal disorder concordant with his symptoms. Plain radiographs showed a soft tissue density in the posterior aspect of the right thigh. MRI with gadolinium contrast of the right lower extremity showed a large $(6 \times 7 \mathrm{~cm})$ soft tissue mass encompassing the sciatic nerve. The lesion is hyperintense on T1-weighted fat-saturated (Fig. 2) and short tau inversion recovery (Fig. 3) images. 


\section{Differential Diagnosis}

Spine

Lumbar spinal stenosis

Herniated lumbar disc

Degenerative lumbar spondylolisthesis

Pelvic

Retroperitoneal bleeding

Piriformis syndrome

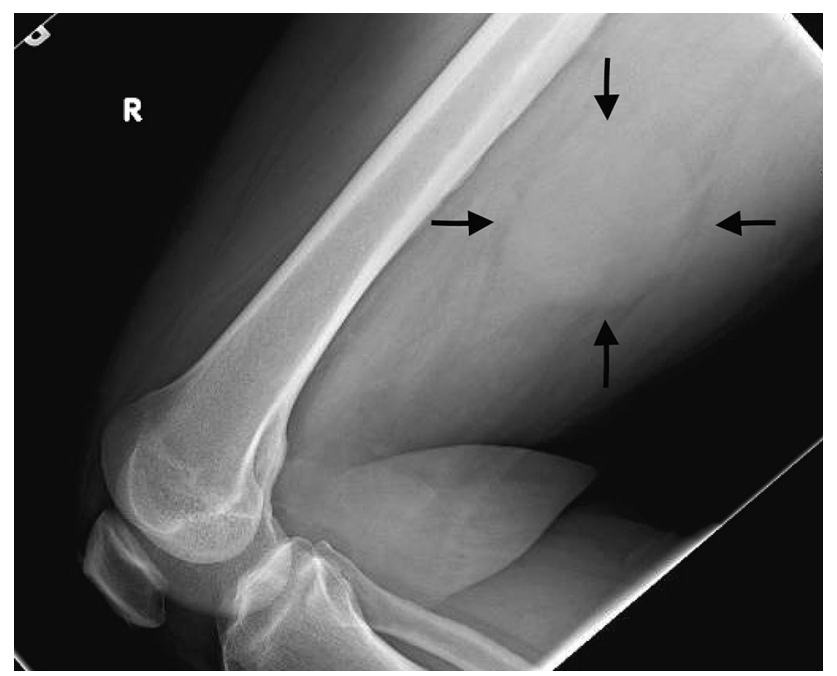

Fig. 1 A radiograph of the lateral femur shows a soft tissue mass in the posterior compartment of the thigh (arrows).

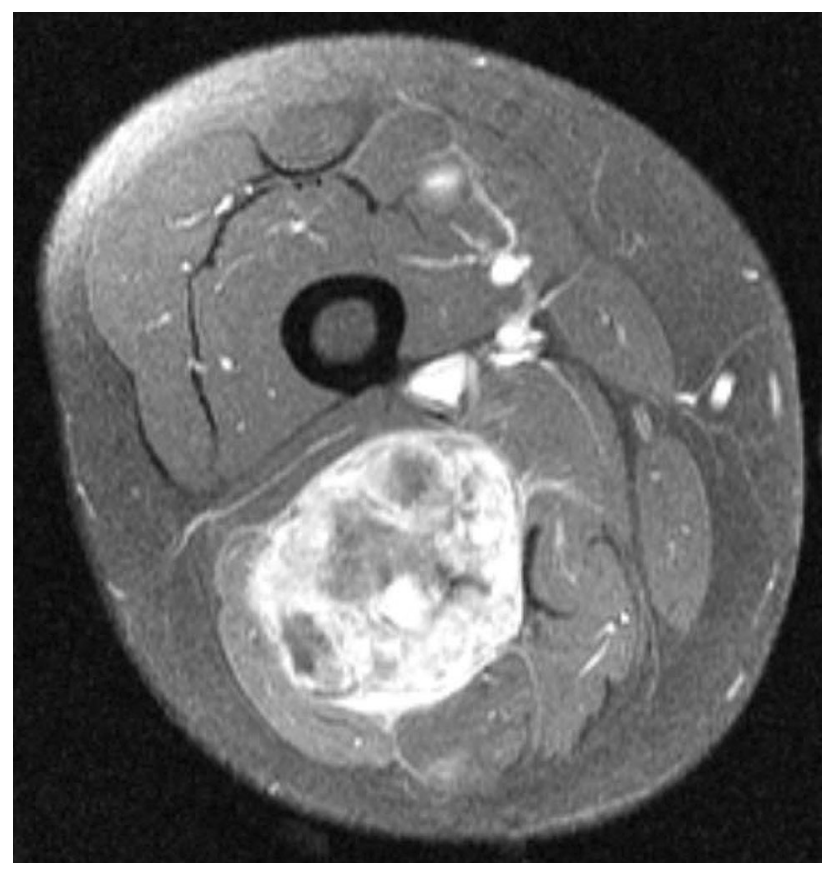

Fig. 2 An axial T1-weighted fat-saturated MR image with gadolinium contrast shows a hyperintense mass in the posterior compartment of the thigh, originating from the sciatic nerve.
Tumor

Intraneural

Schwannoma

Intraneural perineurioma

Neurofibroma

Neurolymphomatosis

Malignant peripheral nerve sheath tumor (MPNST)

\section{Compressive}

Leiomyosarcoma

Rhabdomyosarcoma

Lipoma

Extraosseous Ewing sarcoma

Metastasis

Based on patient history, physical examination, and imaging studies, what is the diagnosis and how should the patient be treated?

\section{Histology Interpretation}

An ultrasound-guided biopsy of the posterior thigh lesion was performed. Pathologic evaluation of the aspirated material showed malignant small, round, blue cells with necrosis (Fig. 4) that were immunoreactive for CD99. Fluorescence in situ hybridization was positive for the EWSRl t(11;22) translocation (Fig. 5).

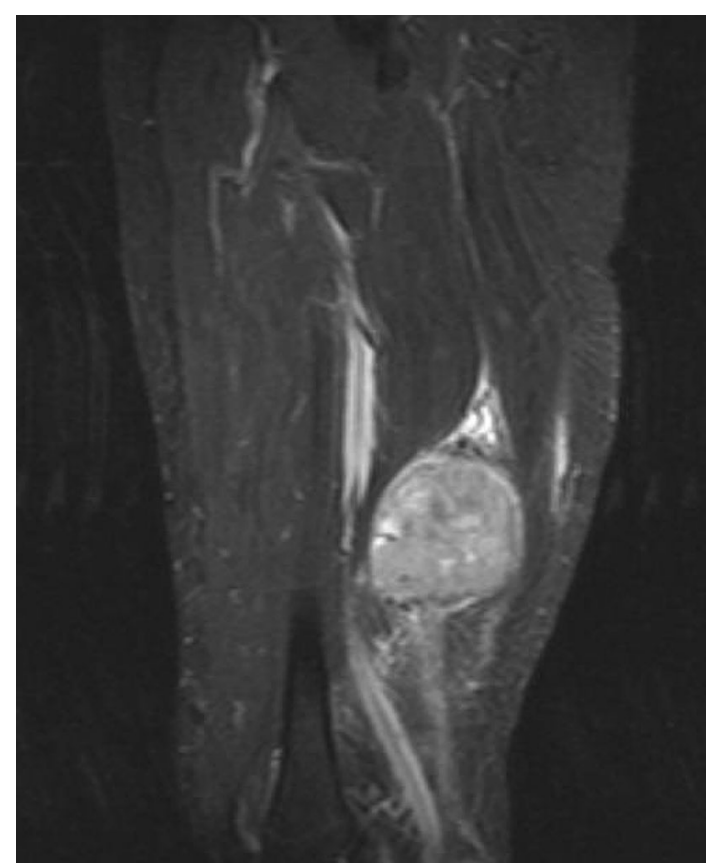

Fig. 3 A sagittal short inversion time recovery MR image shows a hyperintense mass in the posterior compartment of the thigh, originating from the sciatic nerve. 


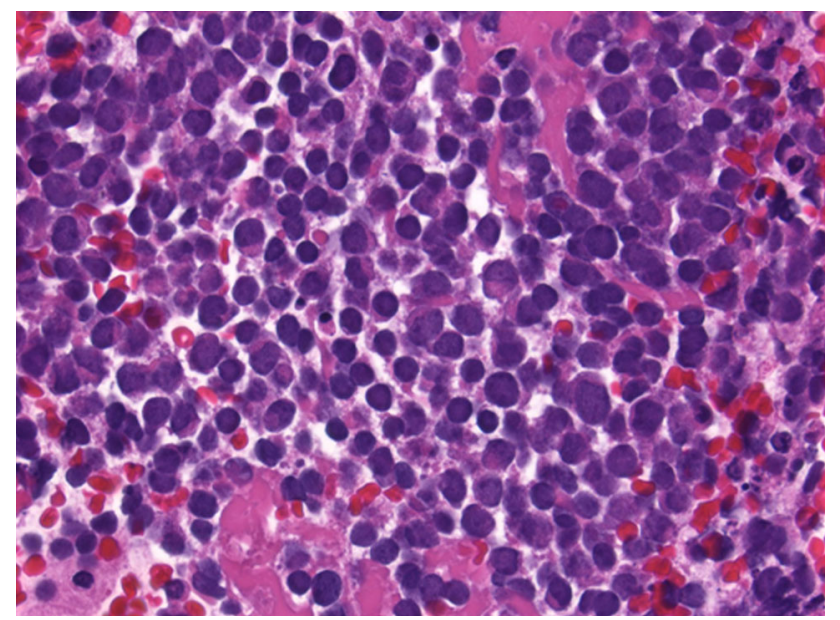

Fig. 4 The fine-needle aspirate shows malignant, blue, round cells (Stain, hematoxylin \& eosin; original magnification, $\times 600$ ).

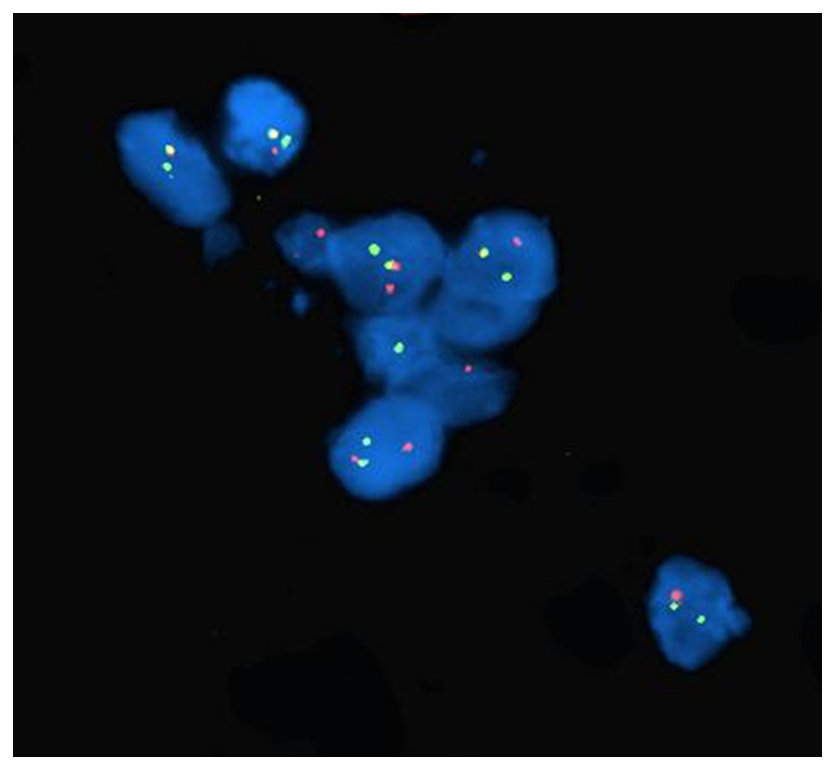

Fig. 5 The fluorescence in situ hybridization shows a translocation of the EWSR1 gene at $22 \mathrm{q} 12$ in tumor cells.

\section{Diagnosis}

Extraosseous Ewing sarcoma.

\section{Discussion and Treatment}

Low back pain and radiculopathy are among the most common symptoms that bring patients to emergency rooms, primary care clinics, and neurosurgical and orthopaedic surgery clinics. The most frequent causes of these symptoms are herniated nucleus pulposus, lumbar spinal stenosis, and degenerative spondylolisthesis. A majority of low back pain
(90\%) and radiculopathy will resolve with time without any surgical intervention or need for advanced imaging. Most patients can be treated without surgery, using NSAIDs, physical therapy, epidural steroid injections, and/or activity modifications. When low back pain and radiculopathy persist despite nonsurgical approaches, further evaluation and imaging are indicated. However, spinal MRI is extremely sensitive and has been shown to display radiographic findings in $50 \%$ of people without clinical low back pain or radiculopathy [9]. If symptoms persist, spine surgery may be indicated if the etiology is spinal, as it most commonly is. However, clinical correlation is critical; with our patient, learning that he had been treated for a concurrent hamstring strain prompted the discovery of a large mass in his posterior thigh on examination. Thus, we were provided with an important clue that directed our imaging to the lower extremity. Tissue sampling of the tumor then was obtained for histologic evaluation. Results showed malignant, small, round, blue tumor cells with necrosis with immunoreactivity for CD99. Fluorescence in situ hybridization also was positive for the EWSRI translocation, confirming our diagnosis of extraosseous Ewing sarcoma (Fig. 5).

The differential diagnoses in this case can be systematically divided to spinal, pelvic, and appendicular causes. The most common causes of lower extremity radiculopathy originating from the spine include lumbar spinal stenosis, herniated lumbar disc(s), and degenerative lumbar spondylolisthesis. These symptoms develop from nerve root impingement and physical examination findings often follow specific nerve root distributions that were not present in this patient. Furthermore, MRI of the spine did not reveal anatomic pathologic features, making these diagnoses less likely.

Extraspinal causes of radiculopathy have been described in large retrospective reviews [10,11]. Pelvic causes of radicular pain may arise from extrinsic compression of the lumbosacral plexus as it traverses the true pelvis or as the L3-S4 nerve roots coalesce to form the sciatic nerve before exit through the greater sciatic foramen. Piriformis syndrome causes sciatic neuropathy in the foramen and symptoms are exacerbated with provocative maneuvers such as flexion, adduction, and internal rotation. Plain radiographs and MR images typically are normal. As such, based on the physical examination this diagnosis is less likely. Similarly, retroperitoneal bleeding may cause radicular signs and symptoms. Patients typically present with hypotension and serologic abnormalities in the setting of trauma or coagulopathy, which were not evident in this case.

Appendicular causes of extraspinal radiculopathy from peripheral nerve tumors have been described [11]. Intraneural tumors may arise from connective tissue such as the perineurium, from myelin-producing Schwann cells, or from peripheral nerve infiltration by lymphoma or nontumor lymphocytes. Physical examination often is consistent 
with a palpable mass as with our patient, therefore, biopsy and pathologic evaluation are critical for diagnosis and guidance with therapy. Schwannoma (or neurilemoma) is the most common peripheral nerve tumor in adults which can affect motor and sensory nerves. The tumor is well encapsulated and histologic analysis shows Antoni A and B structures with pathognomonic Verocay bodies, none of which were present in this case. Intraneural perineurioma is a benign, painless, and slowly progressive tumor associated with loss of motor and sensory function in the affected nerve. Histologic analysis typically shows pseudo-onion bulbs with concentric intraneural lamellar proliferations of perineural cells and immunohistochemistry positive for epithelial membrane antigen and S100 protein, both of which were negative in our case. Neurofibromas arise from nonmyelinating Schwann cells and may be found in young patients with type I neurofibromatosis or later in life if sporadic. Our patient did not have characteristic physical examination findings consistent with type I neurofibromatosis such as café-au-lait spots or axillary freckling. Histologic analysis of his biopsy specimen did not show fibroblast predominance with elongated, wavy nuclei and therefore was inconsistent with this diagnosis. Neurolymphomatosis is a rare intraneural tumor caused by direct spread of non-Hodgkins lymphoma or by a paraneoplastic mechanism to the proximal nerve roots. This entity is found in patients with widespread non-Hodgkins lymphoma and may be the first manifestation of relapse, both of which were not found in our patient. Finally, a malignant peripheral nerve sheath tumor (MPNST) often presents as a large palpable mass on peripheral nerves. In contrast to our patient, these tumors are hypointense on $\mathrm{T} 1$-weighted and hyperintense on
T2-weighted MR images and pathologic analysis reveals spindle cells with wavy nuclei and S100 positivity.

Soft tissue tumors may cause extraspinal radiculopathy through an extrinsic compressive mechanism. Leiomyosarcoma arises from the smooth muscle cells lining blood vessels. These tumors can present in the soft tissues or as intramedullary osteolytic lesions in the metaphysis of long bones that may extend into the soft tissues and cause nerve compression. T2-weighted MR images would reveal a heterogeneous mass with areas of hyperintensity and pathologic features consistent with actin and vimentin immunoreactive cells, not present in our case. Rhabdomyosarcoma is the most common sarcoma in children, but alveolar and pleomorphic subtypes can occur in adults. Immunohistochemistry in these malignant tumors is not consistent with our case and typically features strong positivity to MyoDl, myoglobin, myosin, desmin, and vimentin. Similarly, lipomas, which are benign tumors of mature adipocytes, can cause extrinsic nerve compression but histologic analysis would reveal a bland, hypocellular stroma consistent with normal adipose tissue. Metastasis was ruled out in our case by imaging and pathologic evaluation, but malignant tumors can cause soft tissue metastases. Examples to be considered include lung cancer and melanoma.

Ewing sarcoma is a well-known, primary malignant bone tumor that first was described by James Ewing as an "endothelioma of bone" in 1921 [5]. We report here on a patient with extraosseous Ewing sarcoma, which has been described by others [1]; however, an extraosseous Ewing sarcoma originating from peripheral nerves is extremely rare and has been described in only a few case reports $[6,7,13,14]$. Typically, a Ewing sarcoma is a neoplasm that occurs in children and adolescents, most often patients are in the second
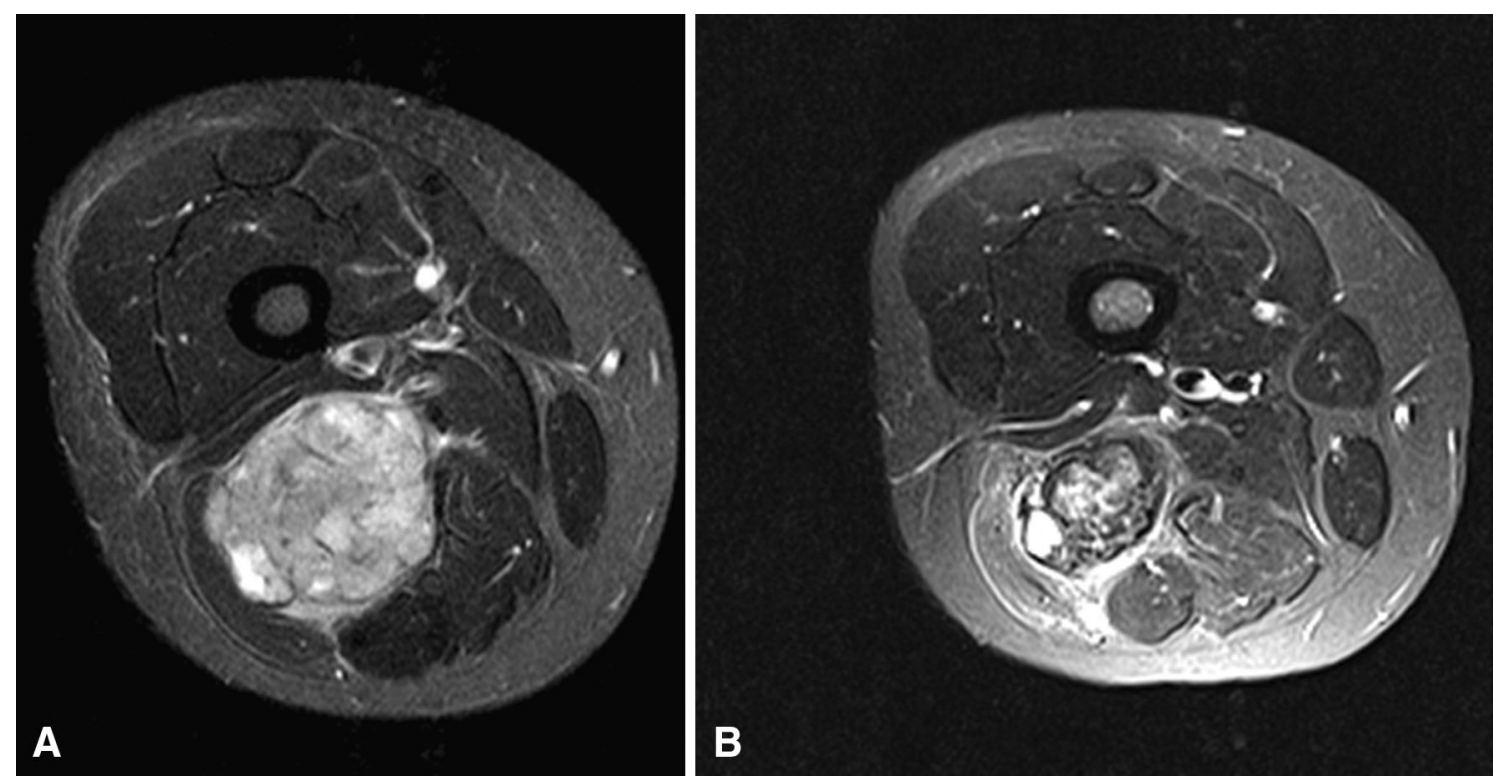

Fig. 6A-B The T2-weighted axial MR images of the right thigh (A) before and (B) after chemotherapy are shown. 


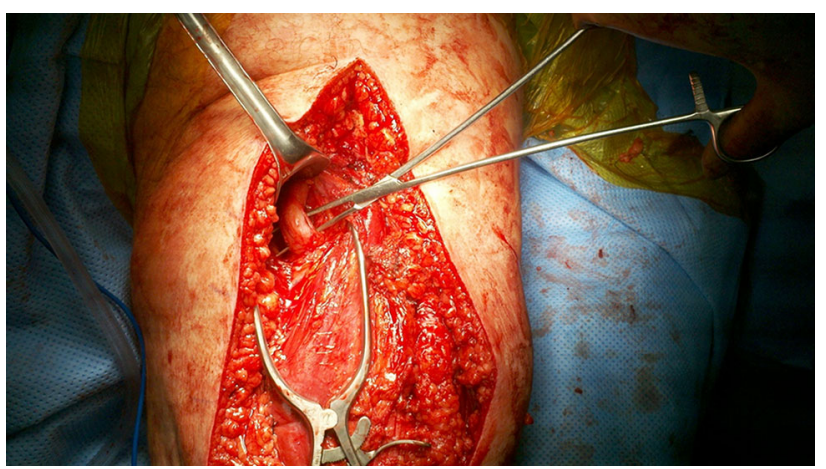

Fig. 7 Intraoperative isolation and surgical amputation of the sciatic nerve was performed.

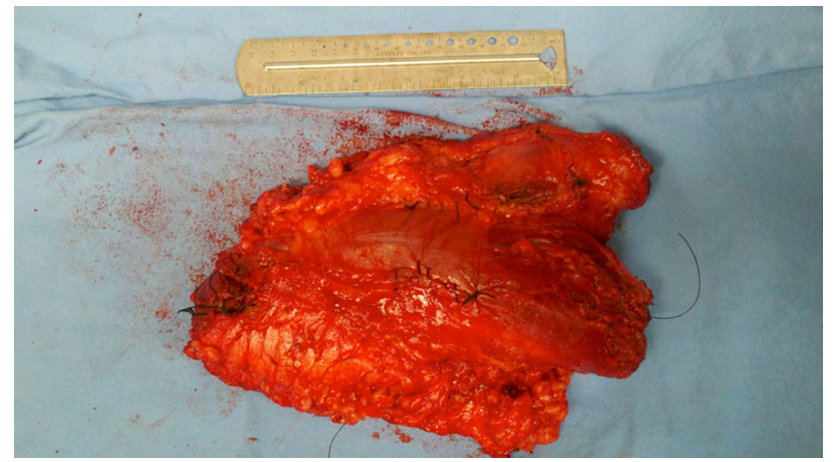

Fig. 8 The intraoperative en bloc resection of the extraosseous Ewing sarcoma of the sciatic nerve is shown.

decade of life with fewer than $20 \%$ of all cases occurring after the age of 20 years [8]. However, rare cases of Ewing sarcoma have been reported even in elderly patients as late as in the ninth decade of life [12]. Typical clinical presentation of Ewing sarcoma includes pain, swelling, fever, and leukocytosis [3]. Pathologically, Ewing sarcoma belongs to the small, round, blue cell family of tumors, which includes neuroblastoma, medulloblastoma, rhabdomyosarcoma, retinoblastoma, non-Hodgkins lymphoma, Wilm's tumor, and primitive neuroectodermal tumor, which is closely related to Ewing sarcoma. Owing to the large number of diagnoses associated with a similar cytologic appearance, it is imperative to make the correct diagnosis because of the great differences in treatment and prognosis. Pathologists use myriad techniques, including immunohistochemistry (CD99, synaptophysin) and cytogenetic analyses, to evaluate for the presence of the classic $\mathrm{t}(11 ; 22)$ Type 1 translocation associated with $90 \%$ to 95\% of cases of Ewing sarcoma [2, 4, 15-17]. Once the diagnosis has been confirmed as Ewing sarcoma, the treatment regimen consists of neoadjuvant chemotherapy, surgical resection, and consolidation chemotherapy. External-beam radiotherapy is reserved for specific indications, which lie beyond the scope of this manuscript.
In our case, staging CT scans of the chest, abdomen, and pelvis were negative. The patient was treated with three preoperative sessions of (vincristine, ifosfamide, doxorubicin, and etoposide [VIDE]) chemotherapy and repeat MRI showed a decrease in tumor size to $4 \times 4 \mathrm{~cm}$ (Fig. 6). The patient was taken to the operating room 4 weeks after his last chemotherapy treatment. A posterior thigh approach was developed with sharp dissection, blunt dissection, and electrocautery. The peroneal and tibial nerves were identified, ligated, and transected distal to the tumor, while proximal dissection revealed the sciatic nerve, which was ligated and transected (Fig. 7). The tumor was circumferentially dissected free of the surrounding tissues and delivered en bloc from the field. The specimen had mobile tissue circumferentially around the tumor, suggesting negative margins (Fig. 8). Pathological analysis of the specimen showed negative surgical margins, extensive tumor necrosis, and marked fibrosis encompassing the sciatic nerve. The patient's postoperative course, including two cycles of adjuvant VIDE treatment and four cycles of vincristine, actinomycin, and ifosfamide, was uneventful and the patient regained hamstring function with expected ankle plantar and dorsiflexion absence. He remains free of local recurrence and metastatic disease at last followup, 19 months after the index procedure and is back to work as a laborer. He wears an ankle-foot orthosis brace but has no other assistive devices.

\section{References}

1. Arpornchayanon O, Hirota T, Itabashi M, Nakajima T, Fukuma H, Beppu Y, Nishikawa K. Malignant peripheral nerve tumors: a clinicopathological and electron microscopic study. Jpn J Clin Oncol. 1984;14:57-74.

2. Aurias A, Rimbaut C, Buffe D, Dubousset J, Mazabraud A. [Translocation of chromosome 22 in Ewing's sarcoma] [in French]. C R Seances Acad Sci III. 1983;296:1105-1107.

3. Dahlin DC, Coventry MB, Scanlon PW. Ewing's sarcoma: a critical analysis of 165 cases. J Bone Joint Surg Am. 1961;43:185-192.

4. Downing JR, Head DR, Parham DM, Douglass EC, Hulshof MG, Link MP, Motroni TA, Grier HE, Curcio-Brint AM, Shapiro DN. Detection of the (11;22)(q24;q12) translocation of Ewing's sarcoma and peripheral neuroectodermal tumor by reverse transcription polymerase chain reaction. Am J Pathol. 1993;143:1294-1300.

5. Ewing J. The Classic: Diffuse endothelioma of bone. Proceedings of the New York Pathological Society. 1921;12:17. Clin Orthop Relat Res. 2006;450:25-27.

6. Isefuku S, Seki M, Tajino T, Hakozaki M, Asano S, Hojo H, Hatori M. Ewing's sarcoma in the spinal nerve root: a case report and review of the literature. Tohoku J Exp Med. 2006;209:369-377.

7. Ishikawa S, Ohshima Y, Suzuki T, Oboshi S. Primitive neuroectodermal tumor (neuroepithelioma) of spinal nerve root: report of an adult case and establishment of a cell line. Acta Pathol Jpn. 1979;29:289-301.

8. Iwamoto Y. Diagnosis and treatment of Ewing's sarcoma. Jpn J Clin Oncol. 2007;37:79-89. 
9. Jensen MC, Brant-Zawadzki MN, Obuchowski N, Modic MT, Malkasian D, Ross JS. Magnetic resonance imaging of the lumbar spine in people without back pain. N Engl J Med. 1994;331:6973.

10. Khoury JD. Ewing sarcoma family of tumors. Adv Anat Pathol. 2005;12:212-220.

11. Kleiner JB, Donaldson WF 3rd, Curd JG, Thorne RP. Extraspinal causes of lumbosacral radiculopathy. J Bone Joint Surg Am. 1991;73:817-821.

12. Le Manac'h AP, Rousselet MC, Massin P, Audran M, Levausseur R. Extraspinal sciatica revealing late metastatic disease from parotid carcinoma. Joint Bone Spine. 2010;77:64-66.

13. Mohan AT, Park DH, Jalgaonkar A, Alorjani M, Aston W, Briggs T. Intra-neural Ewing's sarcoma of the upper limb mimicking a peripheral nerve tumour: a report of 2 cases. J Plast Reconstr Aesthet Surg. 2011;64:e153-156.
14. Strom T, Kleinschmidt-Demasters BK, Donson A, Foreman NK, Lillehei KO. Rare nerve lesions of non-nerve sheath origin: a 17-year retrospective series. Arch Pathol Lab Med. 2009;133:1391-1402.

15. Turc-Carel C, Aurias A, Mugneret F, Lizard S, Sidaner I, Volk C, Thiery JP, Olschwang S, Philip I, Berger MP, et al. Chromosomes in Ewing's sarcoma: I. An evaluation of 85 cases of remarkable consistency of t(11;22)(q24;q12). Cancer Genet Cytogenet. 1988; $32: 229-238$.

16. Turc-Carel C, Philip I, Berger MP, Philip T, Lenoir GM. Chromosome study of Ewing's sarcoma (ES) cell lines: consistency of a reciprocal translocation $\mathrm{t}(11 ; 22)(\mathrm{q} 24 ; \mathrm{q} 12)$. Cancer Genet Cytogenet. 1984;12:1-19.

17. Weidner $\mathrm{N}$, Tjoe J. Immunohistochemical profile of monoclonal antibody O13: antibody that recognizes glycoprotein p30/32MIC2 and is useful in diagnosing Ewing's sarcoma and peripheral neuroepithelioma. Am J Surg Pathol. 1994;18:486-494. 